\title{
Identifying bottlenecks in the iron and folic acid supply chain in Bihar, India: a mixed-methods study
}

\author{
Amanda S. Wendt ${ }^{1,2^{*}}$ (D), Rob Stephenson ${ }^{3,4}$, Melissa F. Young ${ }^{4}$, Pankaj Verma ${ }^{5}$, Sridhar Srikantiah ${ }^{5}$, \\ Amy Webb-Girard ${ }^{2,4}$, Carol J. Hogue ${ }^{6}$, Usha Ramakrishnan ${ }^{2,4}$ and Reynaldo Martorell ${ }^{2,4}$
}

\begin{abstract}
Background: Maternal anaemia prevalence in Bihar, India remains high despite government mandated iron supplementation targeting pregnant women. Inadequate supply has been identified as a potential barrier to iron and folic acid (IFA) receipt. Our study objective was to examine the government health system's IFA supply and distribution system and identify bottlenecks contributing to insufficient IFA supply.

Methods: Primary data collection was conducted in November 2011 and July 2012 across 8 districts in Bihar, India. A cross-sectional, observational, mixed methods approach was utilized. Auxiliary Nurse Midwives were surveyed on current IFA supply and practices. In-depth interviews $(n=59)$ were conducted with health workers at state, district, block, health sub-centre, and village levels.

Results: Overall, 44\% of Auxiliary Nurse Midwives were out of IFA stock. Stock levels and supply chain practices varied greatly across districts. Qualitative data revealed specific bottlenecks impacting IFA forecasting, procurement, storage, disposal, lack of personnel, and few training opportunities for key players in the supply chain.

Conclusions: Inadequate IFA supply is a major constraint to the IFA supplementation program, the extent of which varies widely across districts. Improvements at all levels of infrastructure, practices, and effective monitoring will be critical to strengthen the IFA supply chain in Bihar.
\end{abstract}

Keywords: Maternal health, Evaluation, Antenatal care, Iron and folic acid, Qualitative, Supply chain

\section{Background}

Worldwide 1.62 billion people are estimated to have anaemia [1]. A recent analysis estimates that anaemia affects $38 \%$ of pregnant women globally [2]. In Bihar, India, even greater proportions of ever-married (60\%) and pregnant women $(58 \%)$ are anaemic, higher than national prevalence estimates $(53 \%$ and $50 \%$, respectively) $[3,4]$. Women with maternal anaemia are more likely to deliver preterm and have low birth weight offspring [5] with low foetal iron stores leading to impaired cognitive development [6]. Severe anaemia can lead to

\footnotetext{
* Correspondence: amanda.wendt@uni-heidelberg.de

'UniversitätsKlinikum Heidelberg, Institut für Public Health, Im Neuenheimer Feld 324, 69120 Heidelberg, Germany

${ }^{2}$ Nutrition and Health Sciences, Division of Biological and Biomedical Sciences, Emory University, 1462 Clifton Rd. Suite 314, Atlanta, GA 30322, USA

Full list of author information is available at the end of the article
}

complications during delivery including increased blood loss, cardiac failure, and maternal mortality [5]. Fortunately, oral iron supplementation has been recognized as an effective prevention and treatment of iron deficiency anaemia $[7,8]$.

In 1970, the Government of India established the National Nutritional Anaemia Prophylaxis Programme to address widespread anaemia, targeting high risk groups for iron supplementation. The programme was expanded in 1991 to universal supplementation of pregnant and lactating women. Current recommendations include a daily dose of $100 \mathrm{mg}$ elemental iron for 100 or more days from 14 to 16 weeks of pregnancy until the 3rd month post-partum [9]. Unfortunately, despite these guidelines, high anaemia prevalence persists [5]. In Bihar, only $10 \%$ of women reported consuming IFA for at least 100 days during their last pregnancy, despite 
$80 \%$ reporting registered pregnancies, implying at least one at least one antenatal care visit [4]. Barriers to IFA intake by pregnant women in India and other low and middle income countries have included gastrointestinal side effects [7, 10-13], lack of comprehensive counselling by healthcare providers [14-17], IFA negatively seen as medicine [14], and distrust of government IFA or freely available IFA $[14,17]$. Supply issues have also been recognized as a barrier to IFA adherence [14, 16-18]. In Bihar, this seems likely as infrastructure, personnel, and supply chain challenges have been highlighted as major constraints of the health system. A 2009 report found Bihar's health sub-centres to be lacking in all existing amenities and functionality indicators measured [19]. In $2007-08$, a state-wide survey found only $56 \%$ of primary health centres and $6 \%$ of sub-centres had at least $60 \%$ of required drugs present. In particular, IFA had been out of stock 10 days of the previous month in $78 \%$ of surveyed health sub-centres [20]. More recent reports from 2014 to 2016 continue to highlight drug shortages and procurement challenges as key issues to address in the Bihar health system [21-23]. To our knowledge, no studies to date have set out to comprehensively describe the IFA supply chain in India, characterize potential bottlenecks to access and availability, and identify feasible and contextually relevant solutions.

Our objective was to explore the current status of the Government of Bihar's IFA supply and distribution system to identify bottlenecks that may contribute to insufficient or inconsistent supply. Based on knowledge gained from participant responses, we offer practical recommendations that can be implemented by the government and collaborating organizations.

\section{Methods}

In November 2011 and July 2012 we conducted a crosssectional, mixed methods study to characterize IFA supply chain protocols and procedures across 8 districts. These were the initial focus districts of a CARE Bihar (a non-governmental humanitarian organization) project titled the Integrated Family Health Initiative conducted with support from the Bill and Melinda Gates Foundation. This programme supports the Bihar government to improve maternal and child health outcomes by increasing delivery, uptake, and utilization of family health services [24]. Our study included qualitative in-depth interviews with key players in the IFA supply chain and surveys distributed to Auxiliary Nurse Midwives (ANMs).

From both qualitative and quantitative data collection, we developed a description of the IFA supply chain and ANM receipt and distribution. From qualitative data, we present supply chain bottlenecks identified by interview participants.

\section{Qualitative in-depth interviews Participants}

In total, 59 in-depth interviews were conducted with health workers at state, district, block, health sub-centre, and village levels. Officials in the Health Department, National Rural Health Mission (NRHM), and Integrated Child Development Scheme (ICDS), were included (Table 1). ANMs, Accredited Social Health Activists (ASHAs), and Anganwadi Workers (AWWs) are classified as frontline workers (FLWs) as they are responsible for IFA distribution to beneficiaries in the field (see Additional file 1, Additional file 2, Additional file 3, Additional file 4, Additional file 5, Additional file 6, Additional file 7 and Additional file 8 for interview guides).

Interviews were requested by CARE staff. Frontline workers were recruited based on a priori selection of coverage areas. Initially, blocks near to and far from the district capital, as defined by CARE staff, were chosen to add variation. Later, blocks were selected randomly with modifications due to time constraints.

\section{Data collection}

In-depth interview guides, drafted in English, focused on respondents' perceived roles in IFA receipt and distribution, IFA need estimation, and trainings. Guides were constructed taking into account relevant literature [16] and iterative discussions with co-authors and CARE personnel. One bilingual research assistant used English guides to conduct interviews. Others required interview guides translated into Hindi. Quality and accuracy were verified through review of guides and recordings by external bilingual researchers. All interviews were conducted in Hindi and lasted 30-60 min. Research assistants were trained in qualitative research techniques and debriefed daily with the primary author. Interviews

Table 1 Qualitative In-Depth Interviews Conducted

\begin{tabular}{llc}
\hline $\begin{array}{l}\text { Supply Chain } \\
\text { Level }\end{array}$ & Participants Interviewed & Total Interviews (n) \\
\hline State & State Health Society Official & 20 \\
District & Civil Surgeon & \\
& District Programme Manager & \\
& District Storekeeper & 14 \\
& District Hospital Manager & \\
Block & Medical Officier in Charge & \\
& Block Health Manager & 13 \\
& Block Community Mobilizer & 11 \\
& Pharmacist \\
& Clerk & \\
Heallage & Auxiliary Nurse Midwife & \\
& Accredited Social Health Activist & \\
& Anganwadi Worker & \\
\hline
\end{tabular}


were recorded along with written notes and observations. The majority of recordings were transcribed directly into English by a bilingual researcher. Ten additional interviews, collected in July 2012, were transcribed into Hindi and translated into English in a two-step process. On a subset of transcripts, quality checks were completed by comparison of recordings to transcripts by an external bilingual researcher.

\section{Data management and analysis}

All transcripts were de-identified for analysis. Deductive and inductive codes were created based on research questions, field notes, and participant responses. A codebook was designed including code definitions, inclusion and exclusion criteria, and examples of code use. Thematic analysis was conducted to identify major themes and supply chain bottlenecks. These were then compared across districts, occupation groups, and supply chain levels (e.g. district, block, health sub-centre, and village). Supply chain bottlenecks were identified throughout the transcripts and compared across groups of respondents to highlight key similarities and differences. Practical recommendations were derived from respondent suggestions, published evaluations of Bihar's drug supply chain, and analysis of the data. Analyses were conducted using MAXQDA (version 10, VERBI Software).

\section{Auxiliary nurse midwife surveys Participants}

ANMs were chosen as target participants because of their dual role as part of the IFA supply chain and key distributors of IFA. ANMs are responsible for their own drug supply management and as the last to receive IFA, are most affected when both district and block shortages occur.

ANMs predominantly work at health sub-centres, which should serve a population of 3000-5000 [25], and coordinate IFA distribution with ASHAs and AWWs, who work at the village level (Anganwadi Centre Population Norm: 400-800) [26]. In Bihar, actual coverage estimates are much larger with health sub-centres serving almost 10,000 [27] and Anganwadi Centres over 1000 [28, 29].

\section{Data collection}

We constructed a 37-item survey in collaboration with CARE staff to assess ANM IFA supply and protocols. We randomly selected 3 ANMs from each of the 137 blocks across 8 districts from lists provided. If an ANM was no longer assigned to that block or refused, then another ANM was randomly chosen. CARE block coordinators, trained on survey contents and overall study objectives, administered surveys to ANMs. Names were not collected to ensure confidentiality (see Additional file 9 for ANM questionnaire).

\section{Data management and analysis}

Trained research assistants input survey data into Excel spreadsheets (Microsoft Excel 2010, Redmond, WA). Quality checks were completed by the primary author.

All analyses were conducted using SAS v9.2 (SAS Inc., Cary, NC, USA). We calculated survey weights to account for non-responses, differing numbers of ANMs per block, and blocks per district. Of the 137 blocks invited to participate, 11 blocks did not respond. Represented blocks $(n=126)$ were compared with nonresponsive blocks $(n=11)$ on 7 antenatal characteristics from CARE programme baseline data. We found these 11 blocks had fewer pregnant women receiving FLW counselling during their last pregnancy on pregnancy danger signs $(p<0.0001)$, emergency preparedness $(p=0.04)$, and family planning $(\mathrm{p}<0.0001)$. We found no significant differences comparing the number of mothers who during their last pregnancy: received at least 90 IFA tablets, were visited two or more times during their last trimester by any FLW, received advice from FLWs on immediate new-born care, or delivered their last child at a health facility (data not shown) (Unpublished data, CARE India).

\section{Ethics}

To maintain confidentiality, all districts were assigned letters (A-H) and health workers are referred to as state, district, and block officials. This was done because for some professions, only one post is occupied in each district. ANMs, AWWs, and ASHAs are referred to by their titles and all storekeepers, including pharmacists and clerks, are referred to as storekeepers.

The Institutional Review Board of Emory University approved this study's protocol, considering it "exempt human subjects research". Local IRB approval was not required as this research was considered part of CARE's program operations, which were conducted with the permission of the Government of Bihar. All respondents provided informed oral consent, which was selected as the research presented minimal risk and a signed consent would be the only document linking the participant to the study.

\section{Results}

Iron and folic acid procurement and distribution

We present here the intended route of IFA from procurement to beneficiary in Bihar (Fig. 1). It should be noted that IFA procurement is largely similar to other medicines listed on the Essential Drug List [30], which are often purchased and distributed together. To our knowledge, there was no singular document which outlined this entire process. However, we gained an understanding of this pathway through interviews, policy documents, and previous research. Lack of clarity and 


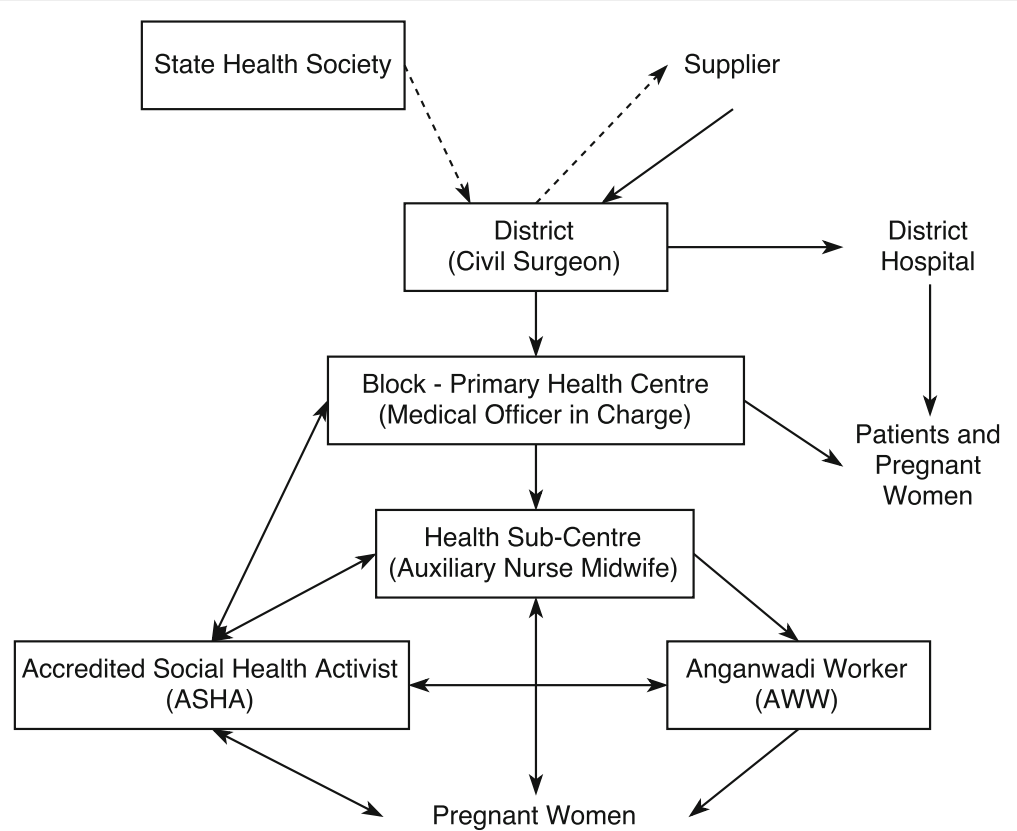

Fig. 1 Major components of the IFA supply chain in Bihar, India. Flow chart showing the distribution of iron and folic acid supplements and funds from the state level (State Health Society) to the beneficiaries (pregnant women). Dotted lines: funds for purchase of IFA supplements; Solid lines: iron and folic acid supplements; Boxes: Surveyed populations; PHC: Primary Health Centre; ANM: Auxiliary Nurse Midwife; ASHA: Accredited Social Health Activist; AWW: Anganwadi Worker

documentation of this process has been cited as a key barrier to successful medicine distribution in Bihar [31]. This has also led to variability in supply chain performance across districts (Fig. 2).

Procurement begins with selection of companies and fixed rates of drugs by the Bihar State Health Society through a competitive bidding process [31-33]. Bihar government documents state that district funding is allocated based on previous consumption [31, 34]. Actual purchasing is decentralized to the district. The Civil Surgeon submits purchase orders based on State Health Society and District Store information, which is then approved by the District Magistrate. This system changed slightly with the establishment of the Bihar Medical Services and Infrastructure Corporation Limited (BMSICL), which centralized drug purchasing to a large extent. Districts now submit purchase orders to BMSICL, who then procures drugs from suppliers and distributes to regional warehouses [35]. As per policy, all districts are to purchase enough drugs for 6 months with a second order after 3-4 months to allow a 2-3 month buffer supply [34]. Districts are responsible for retrieving drugs from Patna-based depots with payment in hand (Cash and Carry) [34]. Drugs should then be distributed to the blocks according to their estimated need (submitted as a written request or 'indent') [34, 36]. Indents

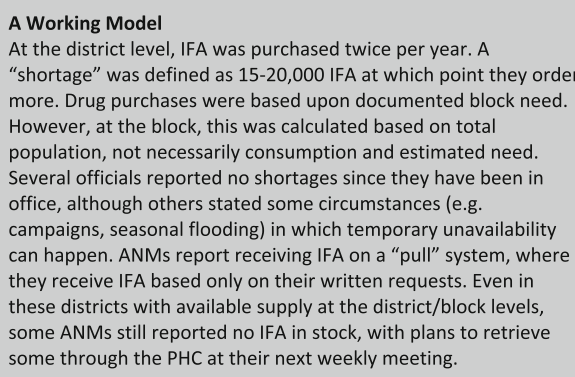

Chronic Shortage

The last time primary health centers and ANMs received IFA was over 6 months ago. This IFA was due to expire the following month; therefore, most of it was thrown away. Beyond that, no IFA had been received in the past year and a half for ANC distribution. One PHC was barely receiving any drugs, despite monthly written requests, "we are only giving B-complex and cough syrup. On that only the whole hospital is running." (Block official). Some ANMs, frustrated hospital is running." (Block official). Some ANMs, frustrated
by the lack of response, have stopped writing drug requests by the lack of response, have stopped writing drug requests the distrust this fostered in patients, who assume that the health workers were stealing their medicines.

Fig. 2 A district comparison. To demonstrate the range of IFA stock and supply chain practices, a brief comparison was made of two districts based on those with a higher and lower functioning IFA supply chain 
from the block store must be approved by the Medical Officer in Charge, the Civil Surgeon, and finally sent to the District Store for fulfilment. From the primary health centre, ANMs receive IFA to distribute at their health sub-centres and monthly Village Health, Sanitation, and Nutrition Days (VHSNDs) [37]. Though IFA distribution and counselling to pregnant and lactating women is clearly outlined [37, 38], there is no clear policy specifically pertaining to health sub-centre drug requests and stock management [34]. At the village level, ASHAs receive IFA independently through ASHA drug kits [39]. AWWs do not receive IFA for antenatal care distribution (Fig. 1). All three are charged with coordinating IFA distribution to pregnant women in their coverage areas through VHSNDs [38]. However, specific roles and stock management between the three positions are not clearly defined.

\section{ANM survey results}

In total, 340 ANM surveys were completed for an $83 \%$ response rate. On average, ANMs reported an average coverage population of 9471 (Table 2), approximately twice the government norm. Only 55.9\% of ANMs reported having IFA in stock on the day of the survey (Table 3). Those that did reported on average 4306 tablets (Table 2). This varied greatly by district from 0 to $90.5 \%$ (Table 3). Few ANMs distributed any IFA to AWWs (21\%) or ASHAs (39\%) in the last month (Table 2).

Almost 10\% of ANMs reported IFA receipt only through fixed deliveries (push-system). Over one-fifth (21.8\%) of ANMs said they only request IFA after they are completely out, meaning they do not maintain a buffer stock (Table 3). Those that did keep a buffer supply reported a wide range of levels with a mean estimate of 751 tablets (Table 3). ANMs who did report using a buffer stock system were more likely to have IFA in stock at the time of the survey (OR: $3.0[1.5,6.0](p=0.0022)$ ).

Table 2 Coverage, iron and folic acid stock, and distribution as reported by ANM, per Health Sub-Centre

\begin{tabular}{lcc}
\hline Characteristic & $\mathrm{n}$ & Mean $(95 \% \mathrm{Cl})$ \\
\hline $\begin{array}{l}\text { Population served by } \\
\text { health sub-centre }\end{array}$ & 288 & $9471(8848,10,094)$ \\
$\begin{array}{l}\text { Pregnant women } \\
\text { registered in previous month }\end{array}$ & 313 & $20(19,22)$ \\
$\begin{array}{l}\text { Current IFA stock } \\
\text { (number of tablets) }\end{array}$ & 331 & $2325(1744,2906)$ \\
$\begin{array}{l}\text { Current IFA stock, if present } \\
\text { (number of tablets) }\end{array}$ & 182 & $4306(3283,5329)$ \\
$\begin{array}{l}\text { Number of IFA tablets given } \\
\text { to AWW in previous month, if given }\end{array}$ & 71 & $1547(899,2195)$ \\
$\begin{array}{l}\text { Number of IFA tablets given } \\
\text { to ASHA in previous month, if given }\end{array}$ & 131 & $619(357,881)$ \\
\hline
\end{tabular}

Extreme values were excluded from each category

ANM Auxiliary Nurse Midwife, IFA iron and folic acid, AWW Anganwadi Worker, ASHA Accredited Social Health Activist
Only $2.9 \%$ of ANMs reported that they could use health sub-centre funds to purchase IFA locally (data not shown) while $67.8 \%$ said they could only receive IFA through the primary health centre (Table 3).

\section{Qualitative data results}

Our analysis of the in-depth interviews revealed several key bottlenecks at various levels in the IFA supply chain.

\section{IFA need}

IFA need was most often reported as calculated based on district size with $3-3.3 \%$ of the total population estimated to be pregnant women. Lactating women, despite being entitled to IFA by policy mandate [9], were not included in this estimate. Some officials emphasized that data from the blocks were not even needed to arrive at this estimate. 'No! We don't need a requirement [from the primary health centres] for placing the order. The number of pregnant women... is calculated. So we place [the order] as per the population' (District F Official).

\section{IFA requests}

IFA purchasing - District According to the state official, suppliers should have medicines ready within 45 days for the first order and 21 days for subsequent orders. To increase supplier accountability to this timeline, the State Health Society established a penalty for late deliveries. 'If they don't deliver on time then from $0.5 \%$ per day up to $37 \%$, I'll deduct their money from the payment' (State Official). However, in almost all districts, drugs were received an estimated 1-3 months after purchase order submission. 'There is no supplier who will supply you the order on time. This problem is always there in the supply chain. If we place an order, we get it after 3 months' (District C Official). 'It takes about one and a half to two months to get the [IFA] delivery' (District A Official). Reporting delayed shipments to the State Health Society was only mentioned in 2 districts. In contrast, most districts did not articulate any strategies to encourage timely drug delivery. 'We just wait for the further supply of the tablets from the company' (District B Official).

Block requests Although the system for requesting IFA was well-known, most health workers did not perceive indents as effective. In District $\mathrm{C}$, a block official did not submit requests based on need alone but called ahead to ask what was available, leading to a lack of documentation of needed drugs which were already out-of-stock. Dependency on the district store was also emphasized, no alternative sources of IFA were perceived to be available in case of district shortages. A block official in District A articulated this frustration with several drugs 
Table 3 ANM reported iron and folic acid supplement supply status and procurement protocols by district

\begin{tabular}{lccccc}
\hline & IFA Out of Stock (\%) & $\begin{array}{c}\text { Cannot Request IFA } \\
\text { from PHC (\%) }\end{array}$ & $\begin{array}{c}\text { Uses Buffer Stock (\%) } \\
\text { from }\end{array}$ & $\begin{array}{l}\text { Average Buffer Stock } \\
\left(\text { mean }(95 \% \text { CI) })^{\mathrm{c}}\right.\end{array}$ & $\begin{array}{c}\text { Only obtains IFA } \\
\text { from the PHC (\%) }\end{array}$ \\
\hline District A & 90.5 & 17.3 & 59.1 & $447(265,628)$ & 81.9 \\
District B & 28.1 & 7.0 & 87.4 & $911(355,1467)$ & 63.2 \\
District C & 63.3 & 5.3 & 81.4 & $544(219,867)$ & 64.1 \\
District D & 0.0 & 7.3 & 92.6 & $1112(520,1704)$ & 81.7 \\
District E & 26.0 & 17.5 & 69.6 & $1196(425,1967)$ & 82.8 \\
District F & 47.8 & 14.3 & 60.2 & $1182(246,2118)$ & 78.1 \\
District G & 16.2 & 3.5 & 82.1 & $303(121,485)$ & 41.0 \\
District H & 13.8 & 19.8 & 42.6 & $1177(613,1743)$ & 82.7 \\
Total & 44.1 & 9.5 & 74.4 & $751(554,949)$ & 67.8 \\
\hline
\end{tabular}

ANM Auxiliary Nurse Midwife, IFA iron and folic acid supplements (100 mg elemental iron and $500 \mathrm{mcg}$ folic acid), PHC Primary Health Centre, Cl

Confidence Interval

${ }^{\mathrm{a} A N M s}$ responded to a multiple choice question asking how they receive IFA from the PHC with 3 possible responses: through fixed deliveries (cannot request), through fixed deliveries (can request), or can only receive IFA through request

${ }^{\mathrm{b}}$ Alternate responses were: request IFA when completely out of stock or cannot request IFA

'Average buffer stock was calculated only among those ANMs reporting buffer stock use

${ }^{d}$ Alternate responses were: can take IFA from ASHA kits, can borrow IFA from other health sub-centres, or can purchase IFA using health sub-centre funds; On average,

$6.9 \%$ of survey data was missing from the above table

which were not being obtained through repeated monthly written requests to the district store.

What will we do? We are helpless. We send the indents regularly to send us folic acid. All basic medicines which are needed to run the hospital, like Paracetamol, Methergine, etc.... even that is not being supplied from the past one and a half years. (District A Block Official)

As indents were not perceived as effective in obtaining drugs, some explained that this procedure was only conducted in case of audits.

Well! There is no result of sending the indent. Whenever they get the IFA they inform us and we get it from them. For our safe side, we send the indent so that no one points out that "you were not having IFA, so what did you do about that?" (District C Storekeeper)

ANM requests We also noted inconsistencies about how ANMs requested IFA from the primary health centre. Block officials reported that ANMs submitted written requests while many ANMs stated that they received IFA from the store without any documentation. Between these fixed deliveries, some ANMs did mention making written or verbal requests if they were out of stock. When the block did not have IFA, ANMs reported that there was no other means to procure IFA for distribution, If there will be no stock at the PHC [primary health centre] then what can we do?' (ANM, District G). All ANMs were also responsible for transport of drugs to their health sub-centre and paid for this at their own expense. One block storekeeper noted that ANMs often refused to take medicines even when available because of transportation difficulties or perceived apathy.

They [ANMs] don't want to take it... we have to make them. At times, they even make excuses like I have not brought my bag this week when I come next week then I will take it. Then they take the medicines afterwards.

(District F Storekeeper)

\section{Buffer stock}

Although officials and storekeepers spoke of maintaining buffer stocks, extra stock kept to avoid stock-outs between purchases, we did not observe buffer stocks in these stores $(n=3)$. All district and block storekeepers who had recently ordered IFA had done so only when they were completely out of stock. In multiple stores, there was little or no IFA, and storekeepers mentioned the futility of writing indents except in case of audit. In other stores, we were told buffer stocks were unnecessary. 'We never felt the need of keeping buffer stock and never faced shortage' (District F Official). Some storekeeping practices also prevented others from maintaining buffer stocks, 'I distribute only to those whose stock gets over' (District F Storekeeper).

\section{Expiring \& expired medicines}

The most common strategy reported to handle expiring drugs (IFA shelf life: 17-23 months) was increased distribution. One storekeeper explained 'We have the pressure of consuming IFA before it gets expired' (District C Block Storekeeper). Another block 
described his attempt at returning about-to-expire drugs to the district.

Then they instruct us to consume the maximum at your level, as there is no need of return, and even it is not done, and to date has not been returned. I cannot even tell you how these medicines are disposed. We just inform the MOIC [Medical Officer In-Charge] and doctor about the status and ask them to prescribe the medicine. (District A Storekeeper)

He further explained that during the most recent incident, expiring stock was distributed in Mahadalit Tolas (the poorest areas). ANMs also reported that excess IFA goes to waste. 'But now we usually get about to expire medicine in bulk from the Civil Surgeon. It is not possible for us to distribute 10,000s of tablets in a month' (ANM, District A).

Stock organization and managing expiration dates was also almost exclusively done either by memory or through the stock register. 'We first keep this in mind, which medicine is of what type, it's all mind talk. The medicines which seem to expire first we supply them first, it stays in my mind' (District F Storekeeper). Only one storekeeper showed us a separate sheet with only expiration dates to quickly find which medicines were about to expire.

\section{Storeroom}

Several district and block officials expressed concern or concessions made because there was not enough space or no fixed location to store medicines. In District A, medicines were stored in district hospital hallways and vacant rooms. Due to this transient space, the storekeeper made a point to distribute medicines quickly as he did not have room to store them. At the block level this trend continued. At one district, the block official and storekeeper mentioned the lack of racks and storeroom disorder.

Regarding ANMs, many told us they stored their medicines with ASHAs, at Anganwadi Centres, or at home because their health sub-centres were not secure. 'We store IFA in our bags with ourselves. The Health Sub-Centre is there but it is not functional so we don't store there' (ANM, District A). Some ANMs did store drugs at their health sub-centre in a locked cupboard or on a rack.

Two storekeepers spoke of rat protection (traps or blocking cracks in the walls) but only one acknowledged the fact that damaged stocks happen.

How much we can avoid the stock from getting spoiled? Rats enter the store and that is the fact. Sometime the medicines even become damaged in transporting or moving it from one store to another store. However much we try, we won't be able to save it. (District G Storekeeper)

\section{Personnel \& training}

District/Block Most health officials did not perceive supply management trainings as helpful.

\section{No! [Trainings are] not given. See, what happens madam is that I don't feel that these kinds of trainings are brain teasing... Once they are in the store they continue with their work. It's not a tough job. The chart is there, you have to fill the serial number and name of the medicine... So filling up these columns is very simple. (District $\mathrm{C}$ Block Official)}

However, at many stores we visited, pharmacists were not even available.

No [I] never received any training, I am a clerk over here, as there is no trained storekeeper or pharmacist. This post is for a pharmacist... but since the government has not arranged for this, still work had to be done so I am doing this. (District A Storekeeper)

Almost all storekeepers said they did not receive supply management training. Only one district hospital official said he received training from the Indian Institute of Health Management Research through the State Health Society. It is possible that this training may have been specific to hospital issues. Both block and district storekeepers explained that their training came from asking others, not from formal supply management training.

'No nothing like that, I have not got any kind of training I just ask from the older people here that what is the requirement.' (District A Block Storekeeper)

'No. I am doing this myself and following the same culture of the person who was working here before me.' (District H Storekeeper)

\section{Health sub-centre/village}

According to training materials, FLWs should be conducting coordinated efforts to identify and register pregnant women, bring them to VHSNDs, distribute IFA, and counsel on IFA consumption and benefits [40]. However interviews revealed varying roles across districts for each FLW. This may be due to inconsistent training across districts concerning IFA distribution. ANMs usually stated that all FLWs distributed IFA. However, it appeared that distribution was often conducted by the ANM and one other FLW. In District H, AWWs were responsible for IFA distribution to beneficiaries and to the ASHAs, ANM gives IFA tablets to me... I give [the ASHA] the tablets.' (AWW, District $\mathrm{H}$ ). ASHAs here did not take a major role in giving IFA, 'I don't distribute much. I write the names of the pregnant 
women who come to the Anganwadi Centre to take the tablets' (ASHA, District H). In contrast, an ASHA in District D explained that AWWs did not distribute IFA, 'No! Apart from the ANM, only ASHA gives [IFA].' However, in other districts, lack of training was a barrier to ASHA IFA distribution. 'ASHAs do not give IFA to pregnant women as they are not confident. So they give the IFA to the ANMs to distribute. The ASHA only brings women to the centre. She does the counselling' (ANM, District A). In another district, ASHAs only distributed under ANM supervision, 'She brings [her IFA] to the centre. They have not been trained properly... When they come to us with their kit, we explain them which medicine to give and to whom' (ANM District D).

\section{Lack of personnel}

The state official mentioned a lack of manpower for the bidding processes in addition to shortages of doctors, pharmacists, and nurses. The lack of Bihar nursing colleges and teachers for ANM training programs were also mentioned as factors. 'There is an acute shortage of doctors and nurses... Here at our ANM schools, there is a shortage of teachers. There are 109 vacancies. For that, only 54 people turned up out of which 34 were placed' (State Official).

\section{Discussion}

IFA supply is a public health issue in Bihar, the extent of which varies greatly across districts. We discovered specific bottlenecks which impacted IFA forecasting, procurement, expired drugs, storage, and an overall lack of personnel. In addition, few training opportunities existed for important players in the supply chain.

In a 2006 comparative analysis, Bossert et al. found that higher performance logistics systems had decentralized planning and budgeting. Conversely, centralization of information systems and inventory control was associated with greater success [41]. The typical scenarios we found in Bihar were in contrast to this ideal scenario. Forecasting future IFA need was usually done using a top-down approach (e.g. calculations from population estimates) rather than based on actual needs. Adjusting orders to fluctuating demand was not possible in most cases because when IFA was unavailable, primary health centre and ANM requests were often not documented. Annual purchasing instead of the recommended 3-4 month timelines also inhibited dynamic forecasting. Though Bihar's inappropriate forecasting mechanisms have been cited as problematic in previous research and reports $[42,43]$, this has usually been seen as a state and district level issue. Our data highlights these issues at the block and health sub-centre level as well. Therefore, to fully address this, changes must also occur at these lower levels to accurately track and react to changing population needs. Similarly, we also found a need for documentation standardization and inventory control. The Bihar government has recognized this need and began implementation to computerize stock information at the district and block levels through BMSICL $[34,44]$. However, this has not been fully rolled out and remains an outstanding issue in current government progress reports [23]. To these strategies, we also recommend increased documentation of subcentre and village levels to more accurately track existing inventories and needs (Table 4).

Improved inventory documentation when fully implemented should bring to light another bottleneck that we found, late supplier deliveries. For this, the obvious strategy is to enforce existing policy as the State Health Society already has penalties in place [44]. Timely reporting is needed from the districts which could be improved through improved communication and process monitoring. This process may have changed substantially as purchasing and delivery are now done directly through BMSICL [44], however reporting of late deliveries remains a potential issue which could affect drug supplies.

As previously discussed, an integral component of successful forecasting is an accurate picture of need. However, we found that many IFA requests were either not being made or recorded. This was due often to convenience or perceptions that these requests were not effective. This occurred particularly during stock-outs, which has been recorded previously $[42,45]$. Training to emphasize the importance of documenting need, especially during times of shortage, as well as an improved response to these requests are both critical to improve this vital part of the supply chain especially at more peripheral levels (Table 4). During prolonged stock-outs, health facilities could implement the use of Rogi Kalyan Samiti (untied) funds for local IFA purchases (Table 4). Use of these funds however, requires a functioning Village Health Nutrition and Sanitation Committee, cooperation between the health sector and panchayat leaders, and awareness of how to spend funds, which have been reported as barriers to fund use in Uttar Pradesh and Maharashtra [46, 47].

We also found that district and block stores were not preparing for stock-outs or late supplier deliveries as evidenced by an overall lack of buffer stocks. Though many storekeepers mentioned buffer stocks and knew their purpose, most did not re-order until completely out of stock. Enforcing current government policy and incorporation of computerized reporting should facilitate appropriate ordering practices, which has been shown to ensure a more consistent drug supply [41] (Table 4). 
Table 4 Identified bottlenecks of Bihar's IFA supply chain, proposed actions, and key stakeholders

\begin{tabular}{ll}
\hline Identified bottleneck & Proposed action \\
\hline $\begin{array}{l}\text { Lack of appropriate IFA } \\
\text { need forecasting }\end{array}$ & $\begin{array}{l}\text { Standardized demand forecasting based on accurate estimates } \\
\text { district needs and previous consumption }\end{array}$ \\
& $\begin{array}{l}\text { Computerization and clear documentation of inventory, stock } \\
\text { requests, and expiry dates }\end{array}$
\end{tabular}

Estimates to include lactating women population; IFA distribution and counseling standard

Late supplier deliveries resulting in inconsistent supply

Indents not being utilized nor perceived as effective

Perceived or actual inability to procure IFA when needed through local purchasing

Lack of buffer stock use at all levels

Utilization of updated BMSICL policy to deduct payment upon late delivery, damaged stock, etc. ${ }^{c}$

Training and monitoring to assure indent use and effectiveness

Explore use of untied funds through Rogi Kalyan Samiti or others to purchase IFA locally in times of shortage

Implementation, monitoring, and evaluation of existing buffer stock requirements ${ }^{\mathrm{d}}$

Ensure adequate storage facilities so stock can be stored safely

No safe disposal plan for expired medicines and pushing of expiring drugs to patients and frontline workers

Storeroom transiency and disorder

Inconsistent training on IFA counseling/distribution across FLW types

Transparent plan to prevent expired medicines through appropriate purchasing practices and safe disposal of expired medicines

Construct, purchase, or long-term rental of adequate storerooms. Funding for racks, labels, and shelves.

Training for storekeepers including storeroom order and inventory protocols.

IFA counseling/distribution training for all frontline workers who work with pregnant women
Key stakeholders

SHS, BMSICL, District Officials ${ }^{a}$, District

Storekeeper, Block Officials ${ }^{b}$, Block

Storekeeper, ANM

SHS, BMSICL, District Officials ${ }^{a}$, District

Storekeeper, Block Officials ${ }^{b}$, Block

Storekeeper, ANM

SHS, BMSICL, District Storekeeper, Block Storekeeper, ANM, ASHA, AWW

SHS, BMSICL, District Officials ${ }^{a}$,

District Storekeeper

SHS, BMSICL, District Officials ${ }^{\mathrm{a}}$, District Storekeeper, Block Officials ${ }^{\mathrm{b}}$, Block Storekeeper, ANM, ASHA

SHS, BMSICL, District Officials ${ }^{a}$, District Storekeeper, RKS, Block Officials ${ }^{b}$, Block Storekeeper, ANM, ASHA

SHS, BMSICL, District Officials ${ }^{a}$, District Storekeeper, Block Officials ${ }^{b}$, Block Storekeeper, ANM, ASHA

SHS, BMSICL, District Officials $s^{a}$, District Storekeeper, Block Officials, Block Storekeeper, ANM

SHS, BMSICL, District Officials ${ }^{\mathrm{a}}$, District Storekeeper, Block Officials ${ }^{\mathrm{b}}$, Block Storekeeper, ANM, ASHA

Training for all frontline workers together at health sub-centre level Block Officials ${ }^{\mathrm{b}}$, ANM, ASHA, AWW to improve coordination and communication
SHS, BMSICL, District Officials $s^{\mathrm{a}}$, District Storekeeper, Block Officials ${ }^{b}$, Block Storekeeper, ANM

SHS, BMSICL, District Officials ${ }^{a}$, District Storekeeper, Block Officials ${ }^{\mathrm{b}}$, Block Storekeeper Block Officials $^{\mathrm{b}}$, ANM, ASHA, AWW 
and communicating their need to the block stores. FLW training on supply management could help to teach basic inventory knowledge (e.g. buffer stocks) and emphasize the use of written requests, to enable accurate documentation of need. Holding trainings through the health sub-centre platform would also allow for coordination and communication between the FLWs, as this would bring together ANMs, ASHAs, and AWWs within a single health sub-centre coverage area [24]. This training should also include IFA distribution to lactating women, as the majority of FLWs we interviewed did not distribute IFA to this population (Table 4).

This study has several strengths. We interviewed a wide range of people at different levels in Bihar's IFA supply chain. Also, in each district we interviewed at least one representative from each level of the supply chain. Through ANM surveys, we also supported our qualitative work by quantitatively demonstrating the variable IFA supply and associated inventory control practices. By utilizing a mixed methods approach, we were not only able to identify bottlenecks along the supply chain but also explore the reasons and justifications for not following certain policies, which would not be possible by conducting quantitative surveys alone.

There were also some limitations to this research. This study is largely qualitative and therefore is not generalizable. However, the 8 districts included were originally selected by CARE as focus districts in order to reflect the state's geographical and political diversity. In addition, we did find that many identified themes agreed with existing supply chain literature from India and other countries. Therefore, bottlenecks identified here may be useful to assess in similar contexts when examining supply chain issues. Though we did talk to several groups involved in IFA receipt and distribution, we did not interview suppliers, drug inspectors, or district magistrates, who also play significant roles in the IFA supply chain. These individuals may provide additional insight into strengths and weaknesses of the current system. Finally, our chosen methodologies were not optimal for detecting the influences of corruption in Bihar's IFA supply chain. Incidences of corruption have been reported in India's health system [46, 49] and in 2014, several officials in BMSICL, which was established to improve drug procurement, were accused of mismanagement in medicine and equipment purchasing. The resulting legal actions halted centralized procurement, causing widespread stock-outs in the state [22, 50, 51]. Further research will be required to understand the impact of corruption on the Bihar supply chain and how good governance practices, such as World Health Organization recommendations [52], can be incorporated into the existing framework.
Previous evaluations of Bihar's essential medicine distribution have also highlighted several bottlenecks identified through this analysis [31, 43, 53]. The creation of the BMSICL [44], was envisioned to address these by streamlining state-level drug procurement and improving documentation protocols. This followed a pattern initiated by several Indian states to emulate Tamil Nadu's drug procurement model, recognized as highly successful $[49,54]$. Such strategies like centralized tendering and purchasing, regional warehouses, and computerized inventory management were planned in order to reduce costs and increase essential medicine accessibility $[44,55]$. In coordination with local organizations, BMSICL has been successful in some of these goals such as warehouse construction, however widespread stock-outs are still being reported [22, 44]. In addition, Bihar has increased IFA purchases to incorporate distribution to lactating women [34], however, according to our data FLWs still do not distribute to this group regularly.

Although the Bihar government is taking steps forward, many of the issues raised in our study may not be resolved by the changes underway. The drug procurement changes focus primarily on the drug tendering process, regional warehouse construction, and improved demand assessment. However, the poor practices we discovered at the district, block, health sub-centre, and village must be addressed to attain a fully-functioning drug supply system. This will become increasingly important in light of the 'Iron+ Initiative', which expands universal IFA supplementation to children from 0 to 19 years and women of reproductive age in addition to pregnant and lactating women. This programme also introduces additional types of IFA tablets, which vary in iron content, size, and color [56]. Especially with the implementation of this programme and existing demands, improving the IFA supply chain will be critical to this initiative's success.

In many iron supplementation programs, low IFA consumption has been assumed to be due to poor adherence, and often attributed to individual factors. However, when the supply chain itself is dysfunctional, this too reduces the value of the intervention in the eyes of the beneficiaries. Inconsistent supply can foster distrust among patients for the healthcare system as a whole [57]. Strengthening the Bihar supply chain will be critical to improving iron supplementation programs in Bihar. More research will be useful to examine how effective monitoring and evaluation can be achieved in addition to the feasibility and maintenance of a more dynamic, flexible supply chain system.

\section{Conclusion}

In this assessment of Bihar's IFA supply, we found that both supply availability and management practices varied depending on the district, especially at the block 
and village levels. Specifically, we discovered issues regarding IFA forecasting, procurement, expired drugs, storage, and an overall lack of personnel. Training on these topics were also often perceived to be unnecessary, and therefore few opportunities existed to change these management practices. Changes are being made at the top levels through reorganization of how stocks are purchased and other measures. But improved practices at the block and village level and improved responsiveness from the state and district will be necessary to truly improve the IFA supply chain in Bihar.

\section{Additional files}

Additional file 1: IDI State official: In-depth interview guide for state level official. (DOCX $20 \mathrm{~kb}$ )

Additional file 2: IDI District official: In-depth interview guide for district officials. (DOCX $19 \mathrm{~kb}$ )

Additional file 3: IDI District storekeeper: In-depth interview guide for district storekeepers. (DOCX $21 \mathrm{~kb}$ )

Additional file 4: IDI Block official: In-depth interview guide for block officials. (DOCX $19 \mathrm{~kb}$ )

Additional file 5: IDI Block storekeeper: In-depth interview guide for block storekeepers. (DOCX 19 kb)

Additional file 6: $|D|$ ANM: In-depth interview guide for Auxiliary Nurse Midwives. (DOCX $20 \mathrm{~kb}$ )

Additional file 7: IDI ASHA: In-depth interview guide for Accredited Social Health Activists. (DOCX $18 \mathrm{~kb}$ )

Additional file 8: $I D \mid$ AWW: In-depth interview guide for Anganwadi workers. (DOCX $18 \mathrm{~kb}$ )

Additional file 9: Questionnaire ANM: Survey administered to Auxiliary Nurse Midwives. (DOCX 18 kb)

\section{Abbreviations}

ANM: Auxiliary Nurse Midwife; ASHA: Accredited Social Health Activist; AWW: Anganwadi Worker; FLW: frontline worker; HSC: health sub-centre; ICDS: Integrated Child Development Scheme; IFA: iron and folic acid; NRHM: National Rural Health Mission; SHS: State Health Society; VHSND: Village Health, Sanitation, and Nutrition Day

\section{Acknowledgements}

The authors would like to acknowledge and thank the study respondents for their time and participation.

\section{Funding}

Funding support was provided by a Bill \& Melinda Gates Foundation grant (\#0000011951) through a subcontract with CARE-India (Cooperative for Assistance and Relief Everywhere) and the Integrated Family Health Initiative in Bihar, India (www.care.org; RM) and T-32 Reproductive, Perinatal, Pediatric Predoctoral National Institutes of Health Grant \#2T32HD052460. The funders had no role in the study design, data collection and analysis, decision to publish, or preparation of the manuscript.

\section{Availability of data and materials}

The dataset used for quantitative analysis is available from the authors. Original interview transcripts used for qualitative analysis will not be shared as informed consent and approval was limited to use of quotes and contextual information to allow interpretation of study findings, while conserving participant anonymity.

\section{Authors' contributions}

AW participated in the study design, created tools and oversaw data collection, conducted analysis and drafted manuscript. RS advised analysis and revised the manuscript. MY assisted and advised study design, data collection and tools, and manuscript revision. PV assisted in data collection and study tool revisions. SS advised regarding study design, study tool revisions and data collection. AWG participated in study design, creation of study tools, and manuscript revision. $\mathrm{CH}$ critically revised the manuscript. UR participated in study design and manuscript revision. RM advised study design, data collection, analysis, and manuscript writing. All authors read and approved the final manuscript.

Ethics approval and consent to participate

The Institutional Review Board of Emory University approved this study's protocol, considering it "exempt human subjects research". Local IRB approval was not required as this research was considered part of CARE's program operations, which were conducted with the permission of the Government of Bihar. All respondents provided informed oral consent, which was selected as the research presented minimal risk and a signed consent would be the only document linking the participant to the study.

\section{Consent for publication}

Not applicable

\section{Competing interests}

The authors declare that they have no competing interests.

\section{Publisher's Note}

Springer Nature remains neutral with regard to jurisdictional claims in published maps and institutional affiliations.

\section{Author details}

${ }^{1}$ UniversitätsKlinikum Heidelberg, Institut für Public Health, Im Neuenheimer Feld 324, 69120 Heidelberg, Germany. ${ }^{2}$ Nutrition and Health Sciences, Division of Biological and Biomedical Sciences, Emory University, 1462 Clifton Rd. Suite 314, Atlanta, GA 30322, USA. ${ }^{3}$ Department of Health Behavior and Biological Sciences, University of Michigan School of Nursing, University of Michigan, 400 North Ingalls Building, Ann Arbor, Ml 48109, USA. ${ }^{4}$ Hubert Department of Global Health, Rollins School of Public Health, Emory University, 1518 Clifton Rd. NE, Atlanta, GA 30322, USA. Integrated Family Health Initiative, CARE Bihar, 2nd Floor, 10, IAS Colony, Kidwaipuri, Patna, Bihar 800 001, India. ${ }^{6}$ Department of Epidemiology, Rollins School of Public Health, Emory University, 1518 Clifton Rd. NE, Atlanta, GA 30322, USA.

Received: 20 July 2016 Accepted: 15 March 2018

Published online: 12 April 2018

\section{References}

1. World Health Organization: Worldwide prevalence of anaemia 1993-2005. In: WHO Global Database on Anaemia. Edited by Benoist B, McLean E, Egli I, Cogswell M. Geneva: World Health Organization; 2008.

2. Stevens GA, Finucane MM, De-Regil LM, Paciorek CJ, Flaxman SR, Branca F, Pena-Rosas JP, Bhutta ZA, Ezzati M. Global, regional, and national trends in haemoglobin concentration and prevalence of total and severe anaemia in children and pregnant and non-pregnant women for 1995-2011: a systematic analysis of population-representative data. Lancet Glob Health. 2013;1 (1):E16-25.

3. International Institute for Population Sciences (IIPS), Macro International. National Family Health Survey (NFHS-4): India fact sheet. Mumbai: IIPS; 2016.

4. International Institute for Population Sciences (IIPS), Macro International. National Family Health Survey (NFHS-4): Bihar fact sheet. Mumbai: IIPSs; 2016.

5. Kalaivani K. Prevalence \& consequences of anaemia in pregnancy. Indian J Med Res. 2009;130(5):627-33.

6. Lozoff B, Jimenez E, Smith JB. Double burden of iron deficiency in infancy and low socioeconomic status: a longitudinal analysis of cognitive test scores to age 19 years. Arch Pediatr Adolesc Med. 2006; 160(11):1108-13.

7. Pena-Rosas JP, De-Regil LM, Dowswell T, Viteri FE. Daily oral iron supplementation during pregnancy. Cochrane Database Syst Rev. 2012;12:CD004736.

8. Imdad A, Bhutta ZA. Routine iron/folate supplementation during pregnancy: effect on maternal anaemia and birth outcomes. Paediatr Perinat Epidemiol. 2012;26(Suppl 1):168-77.

9. Ministry of Health \& Family Welfare. Guidelines for control of Iron deficiency anemia. New Delhi: Government of India; 2013. 
10. Ekstrom EC, Kavishe FP, Habicht JP, Frongillo EA Jr, Rasmussen KM, Hemed L. Adherence to iron supplementation during pregnancy in Tanzania: determinants and hematologic consequences. Am J Clin Nutr. 1996;64(3):368-74.

11. Hyder SM, Persson LA, Chowdhury AM, Ekstrom EC. Do side-effects reduce compliance to iron supplementation? A study of daily- and weekly-dose regimens in pregnancy. J Health Popul Nutr. 2002;20(2):175-9.

12. Mora JO. Iron supplementation: overcoming technical and practical barriers. J Nutr. 2002;132(4 Suppl):853S-5S.

13. Khalafallah AA, Dennis AE. Iron deficiency anaemia in pregnancy and postpartum: pathophysiology and effect of oral versus intravenous iron therapy. J Pregnancy. 2012;2012:630519.

14. Galloway R, Dusch E, Elder L, Achadi E, Grajeda R, Hurtado E, Favin M, Kanani S, Marsaban J, Meda N, et al. Women's perceptions of iron deficiency and anemia prevention and control in eight developing countries. Soc Sci Med. 2002:55(4):529-44.

15. Ghanekar J, Kanani S, Patel S. Toward better compliance with iron-folic acid supplements: understanding the behavior of poor urban pregnant women through ethnographic decision models in Vadodara, India. Food Nutr Bull. 2002;23(1):65-72.

16. Galloway R, McGuire J. Determinants of compliance with iron supplementation: supplies, side effects, or psychology? Soc Sci Med. 1994;39(3):381-90.

17. Nagata JM, Gatti LR, Barg FK. Social determinants of iron supplementation among women of reproductive age: a systematic review of qualitative data. Matern Child Nutr. 2012;8(1):1-18.

18. Sununtnasuk C, D'Agostino A, Fiedler JL. Iron+folic acid distribution and consumption through antenatal care: identifying barriers across countries. Public Health Nutr. 2016;19(4)732-42.

19. Gill K. A primary evalutaion of service delivery under the National Rural Health Mission (NRHM): findings from a study in Andrea Pradesh, Uttar Pradesh, Bihar and Rajasthan. In: Planning Commission of India; 2009.

20. International Institute for Population Sciences (IIPS). District level household and facility survey (DLHS-3), 2007-08: Bihar. Mumbai: IIPS; 2010.

21. Bihar Technical Assistance Support Team (BTAST). Quality improvement efforts in public health facilites of Bihar: some general findings. Patna: BTAST; 2016

22. Sector Wide Approach to Strengthen Health (SWASTH) in Bihar. Annual review - summary. Patna: SWASTH; 2015.

23. National Health Mission (NHM). National health mission programme implementation plan: 2016-17. Patna: NHM; 2016.

24. CARE India: Integrated family health initiative: catalysing change for health communities. 2013.

25. Ministry of Health \& Family Welfare, Government of India. Indian Public Health Standards (IPHS) guidelines for sub-centers: revised 2012. New Delhi: Government of India; 2012.

26. Gangbar J, Rajan P, Gayithri. Working paper 318: Integrated Child Development Services in India - A Sub-National Review. Bangalore: The Institute for Social and Economic Change, 2014.

27. Ministry of Health \& Family Welfare. Rural health statistics in India. New Delhi: Government of India; 2012

28. Ministry of Women \& Child Development. Statewise details of projects, AWC, beneficiaries, vacancy position and nutrition status of children as on March 2011. New Delhi: Government of India; 2011.

29. Pratichi Insitite and Asian Development Institute. The Pratcihi Child Report II: ICDS in West Bengal and Bihar. New Delhi and Patna, India: Pratichi Trust and Asian Development Institute; 2015.

30. Essential Drug List: 2013-4. [http://bmsicl.gov.in/?q=EssentialDrugs]

31. Selvaraj S, Chokshi M, Hasan H, Kumar P: Improving governance and accountability in India's medicine supply system. New Dehli: Public Health Foundation of India; 2010.

32. The Bihar Finance (Amendment) Rules, 2005. [http://finance.bih.nic.in/ Documents/New\%20GFR2005.pdf].

33. Conditionalities compliance for policy of drugs and IT enabled inventory system dt 11.12.13. [http://www.statehealthsocietybihar.org/drugcell/IT_ enabled_inventory_system.pdf].

34. State Health Society, National Rural Health Mission, Government of Bihar: Consolidated revised NRHM state project implementation plan 2012-13 of Bihar. Patna: Government of Bihar; 2011.

35. Padmanathan $P$, Singh M, Mannarath SC, Omar M, Raja S. A rapid appraisal of access to and utilisation of psychotropic medicines in Bihar, India. Int J Ment Heal Syst. 2014;8:29.
36. Directorate General of Health Services, Ministry of Health \& Family Welfare Indian Public Health Standards (IPHS) guidelines for primary health centres. New Delhi: Government of India; 2012.

37. Directorate General of Health Services, Ministry of Health and Family Welfare. Indian Public Health Standards (IPHS) guidelines for sub-Centres. New Delhi: Government of India; 2012.

38. Monthly Village Health Nutrition Day: Guidelines for AWWs/ASHAs/ANMs/ PRIs. In. Edited by National Rural Health Mission, Ministry of Health \& Family Welfare. New Dehli: Government of India; 2007.

39. List of drugs being provided in ASHA kit. [http://www.nhm.gov.in/ communitisation/asha/list-of-drugs-being-provided-in-asha-kit.html].

40. National Rural Health Mission, Ministry of Health \& Family Welfare, Government of India: Monthly village health nutrition day: guidelines for AWWs/ASHAs/ANMs/PRIs. 2007

41. Bossert TJ, Bowser DM, Amenyah JK. Is decentralization good for logistics systems? Evidence on essential medicine logistics in Ghana and Guatemala. Health Policy Plan. 2007;22(2):73-82.

42. Roy C, Das JK, Jha HK, Bhattacharya V, Shivdasani JP, Nandan D. A study on the logistics and supply management system of drugs at different levels in Darbhanga district of Bihar. New Delhi: National Institute of Health and Family Welfare; 2008

43. State Health Society Bihar, Department of Health and Family Welfare, Government of Bihar: State project implementation plan 2011-12. Patna, Bihar, India.

44. Bihar Medical Services and Infrastructure Corporation Limited. [http//bmsicl.govin/].

45. Yadav P: Analysis of the public, private and mission sector supply chains for essential drugs in Zambia. London: DFID Health Resource Centre; 2007.

46. Nandan D, Singh CM, Jain PK, Nair KS, Kumar P, Dhar N. An assessment of utilization of untied fund provided under national rural health mission in Uttar Pradesh. New Delhi: National Institute of Health and Family Welfare; 2009.

47. Sah PK, Raut AV, Maliye CH, Gupta SS, Mehendale AK, Garg BS. Performance of village health, nutrition and sanitation committee: a qualitative study from rural Wardha, Maharashtra. In: The health agenda, vol. 1; 2013. p. 112-7.

48. Directorate General of Health Services, Ministry of Health and Family Welfare Procurement and operation manual for medical store organisation and government medical store depots. New Delhi: Government of India; 2008.

49. Planning Commission of India: High level expert group report on universal health coverage for India. New Delhi: Government of Bihar; 2011.

50. Kumar P. Health for all challenges in Bihar. Indian J Appl Clin Sociol. 2015; 10(4):13-28.

51. Medicine scarcity in Bihar post scam: Modi. In: The Times of India. Patna, Bihar; 2014. https://timesofindia.indiatimes.com/city/patna/Medicine-scarcityin-Bihar-post-scam-Modi/articleshow/42291554.cms.

52. Organization. WH: A framework for good governance in the pharmaceutical sector: GGM model framework. 2008.

53. Roy C, Das JK, Jha HK, Bhattacharya V, Shivdasani JP, Nandan D. Logistics and supply management system of drugs at different levels in Darbhanga District of Bihar. Indian J Public Health. 2009;53(3):147-50.

54. World Health Organization. 4.1 competitive mechanism in public drug supply. In: Selected topics in health reform and drug financying - health economics and drug series, no 006. Geneva: WHO; 1998.

55. Singh PV, Tatambotla A, Kalvakuntla RR, Chokshi M. Replicating Tamil Nadu's drug procurement model. Econ Polit Wkly. 2012;47(39):26-9.

56. Ministry of Health and Family Welfare. Guidelines for control of Iron deficiency Anaemia. New Delhi: Government of India; 2013.

57. Mkoka DA, Goicolea I, Kiwara A, Mwangu M, Hurtig AK. Availability of drugs and medical supplies for emergency obstetric care: experience of health facility managers in a rural district of Tanzania. BMC Pregnancy Childbirth. 2014;14:108. 